\title{
Le métier d'institutrice indépendante francophone à Montréal, 1869-1915
}

\author{
Andrée Dufour \\ Centre interuniversitaire d'études québécoises, Université Laval
}

\section{RÉSUMÉ}

En 2006, nous publiions dans le périodique français Histoire de l'éducation, $\mathrm{n}^{\circ} 109$, un article intitulé « Les premières enseignantes laïques au Québec. Le cas de Montréal, 1825-1835 ». Ces enseignantes, anglophones en majorité, tenaient de petites écoles essentiellement privées dans lesquelles était instruite une importante composante de la jeunesse montréalaise. S'inspirant du modèle implanté par ces récentes immigrantes britanniques, des maîtresses lä̈ques francophones ont suivi cet exemple. Elles vont peu à peu s'approprier ce modèle d'institutrices indépendantes et aussi le poursuivre au XIX ${ }^{\mathrm{e}}$ et même au XX $\mathrm{X}^{\mathrm{e}}$ siècles. Elles ont ainsi participé par leurs écoles, souvent petites, à l'éducation d'une frange significative de la jeunesse notamment au XIX ${ }^{e}$ siècle, et ce, en dépit de l'implantation de la Commission catholique de Montréal en 1846. Aussi nous a-t-il semblé pertinent de nous attarder dans ce texte à l'apport éducationnel de ces enseignantes et ce, plus particulièrement, à celui des années 1869-1915.

\section{ABSTRACT}

In 2006, I published an article, "The first female lay teachers in Quebec. A Montreal Case Study, 1825-1835," in the French journal, Histoire de l'éducation (no. 109). The women teachers that I wrote about, most of whom were anglophones, owned small, essentially private schools in which they taught a significant proportion of Montreal's young population. They inspired francophone female lay teachers who imitated their system, imported recently from Britain. These francophone women would make the model of independent schoolmistress their own, adapting it throughout the nineteenth century, and even into the twentieth. This way, the women contributed through the schools they owned, which were often small, to educating a noteworthy margin of the city's youth, notably in the nineteenth century. They did so despite the founding of the Montreal Catholic School Commission in 1846. Consequently, the present article deals in some detail with the educational contributions of these women teachers in this period, but more specifically between the years 1869 and 1915 . 


\section{Les écoles des « dames et demoiselles » de Montréal, 1869-1915}

Pionnière en la matière, Malvina Marchand, âgée de 25 ans, épouse de feu Médéric Marchand, ouvre à Montréal en 1869 une petite école qui accueille 36 garçons et filles au numéro 13 de la rue Saint-Dominique. La même année, la jeune veuve sollicite un octroi de la Commission scolaire catholique de Montréal ${ }^{1}$. Elle obtient cet octroi étant en quelque sorte une pionnière en ce domaine.

Quelque vingt-cinq ans après sa création, la Commission procède donc de deux façons à l'édification d'un réseau d'écoles primaires pour les enfants catholiques de la métropole. D'une part, elle consacre la majorité de ses revenus à la construction rapide de spacieuses écoles de garçons, dont elle confie la charge à des enseignants laïques. D'autre part, afin de répondre aux demandes pressantes pour que soient créées de nouvelles écoles qui répondraient à la croissance démographique de Montréal ${ }^{2}$, les commissaires accordent aussi des subventions à des congrégations d'hommes et de femmes ainsi qu'à des institutrices läques pour les aider à maintenir des écoles dans les lieux où les besoins se font le plus vivement sentir. La deuxième formule prend de l'ampleur à la fin des années 1870 alors que le Bureau des commissaires subit les contrecoups de la crise économique qui sévit durement à Montréal. Incapable de rencontrer le lourd déficit qu'il a contracté - son administration est même soumise à de vives attaques qui entraîneront la tenue, en 1882, d'une Commission royale d'enquête -, le Bureau doit suspendre toute construction nouvelle et mettre plutôt l'initiative privée au service de l'enseignement primaire public ${ }^{3}$.

En 1886, le Bureau trace un bilan de ses activités dans sa première Notice officielle. Il rapporte alors l'existence d'une trentaine d'écoles placées «sous son contrôle ", soit sous son autorité. En réalité, les commissaires ne sont propriétaires que de six écoles publiques de garçons, les vingt-six autres étant toutes des maisons indépendantes recevant des subventions du Bureau. Quatre d'entre elles sont dirigées par les Frères des Écoles chrétiennes, neuf par des communautés de femmes dont sept par la Congrégation de Notre-Dame et dix par des " dames laïques "4. Ces enseignantes laïques, bien présentes dans l'enseignement dès 1825 , jouent donc encore à la fin du XIX ${ }^{\mathrm{e}}$ siècle un rôle important dans l'instruction des enfants catholiques de Montréal.

La Notice de 1886 indique que la participation des laïques se distingue de celle des religieuses. Ne disposant pas des ressources des communautés de femmes qui sont en mesure de construire des écoles dont elles conservent la propriété, ces laïques doivent installer leurs classes dans des appartements qu'elles louent dans des maisons d'habitation. Par ailleurs, elles reçoivent une clientèle mixte, contrairement aux religieuses qui n'accueillent que des filles ${ }^{5}$, des personnes " du sexe " comme on l'écrivait souvent à l'époque. Enfin, à l'exception de l'école tenue par Madame Marchand, les «dames laïques » dirigent des établissements de taille modeste. La fréquentation et l'assistance y sont également plus faibles que dans les écoles congréganistes. Le ratio professeurs/élèves demeure élevé cependant, ce qui atteste de la lourde tâche assumée par ces institutrices ${ }^{6}$. 
Ces institutrices sont des célibataires. Tout au long de la période étudiée, elles assument la direction de la majorité des écoles laïques subventionnées, que l'on désignera d'ailleurs bientôt comme les écoles des " dames et demoiselles ». La commission scolaire catholique en subventionne entre dix et douze au tournant du siècle. Plusieurs des directrices en fonction sont assistées par des membres de leur famille. Certaines travaillent avec l'aide de leur fille; d'autres, plus nombreuses, en compagnie d'une ou de deux de leurs sœurs. On retrouve également les membres d'une même famille dans différentes écoles des « dames et demoiselles ». Installées dans des locaux rudimentaires, fonctionnant avec un minimum de personnel au sein duquel existent en outre plusieurs liens de parenté, celles-ci fonctionnent en somme comme des entreprises familiales.

Ainsi que l'a souligné la regrettée Marta Danylewycz, le travail représente pour plusieurs une réalité nécessaire et souvent essentielle : certaines célibataires sont obligées d'assurer le gagne-pain de leurs parents, alors que les veuves doivent subvenir aux besoins de leur famille ${ }^{7}$. Malvina Marchand était bien au fait des lourdes responsabilités financières et familiales qui incombaient à un bon nombre de femmes engagées dans l'enseignement. Une nécrologie publiée en 1907 dans La Revue canadienne rapporte qu'elle "voulait que ses élèves fussent prêtes à la lutte pour la vie, à se subvenir à elles-mêmes, à aider quelques vieux parents usés à la tâche, à remplacer le chef de famille, à gagner pour tous au besoin $»^{8}$.

D'autre part, il semble que pour un grand nombre de directrices laïques, l'enseignement primaire représentait plus qu'un indispensable gagne-pain. Leur persévérance en fait foi. Un rapport transmis en 1891 par les commissaires d'écoles au Département de l'instruction publique révèle, en effet, que sur un total de dix directrices, deux sur sept ont obtenu leur brevet d'enseignement au cours des années 1850 et 1860 , une en 1870 et les deux dernières au début des années $1880^{\circ}$. Certes, l'année d'obtention du brevet ne correspond pas nécessairement à la date d'entrée dans l'enseignement. La présence de ces femmes à la tête d'écoles à la toute fin du $19^{\mathrm{e}}$ siècle, présence qui se poursuivra au début du $20^{\mathrm{e}}$ siècle, suggère malgré tout qu'elles étaient déterminées à faire carrière dans l'enseignement. En fait, c'est la mort qui vient interrompre les activités de plusieurs d'entre elles. En 1906, par exemple, Malvina Marchand succombe après avoir consacré 37 ans à la bonne marche de son école, qui porte maintenant le titre d'académie. L'année suivante, c'est au tour de Laure Cronin, qui a accumulé plus de 47 années de service à la Commission scolaire.

Pour ces deux femmes et leurs consœurs, diriger une école subventionnée représente un véritable défi, car plusieurs conditions sont rattachées à l'obtention d'un octroi de la Commission scolaire. Les directrices doivent d'abord faire approuver et recommander leur école par le curé de la paroisse ${ }^{10}$. Les commissaires considèrent ensuite si celle-ci répond, en raison de son emplacement, aux besoins de leur collectivité. De ceci découle l'interdiction, pour les directrices ayant reçu un octroi, de déplacer leur école sans avoir obtenu l'autorisation de la Commission scolaire. Enfin, ces dernières sont tenues de souscrire aux règlements pédagogiques et hygiéniques prescrits par le Comité catholique du Conseil de l'Instruction publique et par les autorités sanitaires provinciales et municipales, de dispenser le programme d'études 
autorisé par la Commission scolaire et de n’utiliser que les manuels sélectionnés par les commissaires ${ }^{11}$. Un grand nombre d'enseignantes laïques propriétaires d'une école privée s'empressent malgré tout de solliciter un octroi des commissaires. Ceux-ci rejettent plusieurs requêtes en invoquant l'insuffisance des revenus à leur disposition. Quant aux écoles pour lesquelles les enseignantes laïques réussissent à décrocher une subvention, elles fonctionnent avec de maigres ressources. Les sources disponibles ne fournissent pas de renseignements précis sur la façon dont sont répartis les octrois entre les différentes écoles laïques subventionnées durant les années 1870 et 1880 . Toutefois, en 1880-1881, le Bureau des commissaires leur verse entre 254 et 700 \$, montant qui comprend les salaires des institutrices. L'octroi le plus élevé est accordé à l'école de Madame Marchand. Le coût annuel par élève s'échelonne de 1,36 \$ à 2,05\$. Pourtant, la même année, les dépenses totales encourues pour les écoles laïques de garçons "directement sous contrôle " ${ }^{12}$ se situent entre $5625 \$$ et $13515 \$$, le coût annuel par élève variant de $12,05 \$$ à $18,91 \${ }^{13}$.

Au milieu des années 1890, le Bureau adopte un mode de financement uniforme pour l'ensemble de ses écoles subventionnées, y compris celles dirigées par des enseignantes laïques. Désormais, la subvention annuelle sera proportionnelle au nombre d'élèves fréquentant l'école, pourvu que celui-ci n'excède, dans aucune des classes, le nombre fixé par les autorités sanitaires. La fréquentation sera calculée sur la base de l'assistance moyenne enregistrée durant les cinq premiers mois de l'année scolaire. Dans le cas où elle jugerait cette dernière insuffisante, la Commission pourra diminuer ou même supprimer l'octroi. Celui-ci est fixé à 3 \$ par élève pour les écoles dirigées par des institutrices, tandis que les maisons dirigées par des instituteurs reçoivent 6 \$ par élève. Le Bureau des commissaires n’assume en aucun cas la responsabilité du loyer, de l'ameublement et des dommages qui peuvent être causés à la propriété occupée par une école subventionnée. Il concède plutôt aux directeurs et aux directrices le droit de conserver le produit de la rétribution mensuelle perçue des enfants, afin de les aider à couvrir les frais d'entretien, d'éclairage et de chauffage de l'école ${ }^{14}$.

Les commissaires aident à l'occasion les directrices laïques à s'installer dans un meilleur local ou à améliorer l'état de celui qu'elles occupent. Cependant, la plupart des directrices sont tracassées, tout au long de la période étudiée, par de nombreux embarras financiers. Aussi revendiquent-elles sans cesse une subvention plus élevée. Elles le font à titre individuel mais, de plus en plus aussi, de façon collective, ce qui atteste de la croissance d'un esprit de corps parmi ces dernières. De fait, leurs multiples interventions amènent les commissaires à augmenter graduellement l'octroi qu'ils leur consentent. Celui-ci atteint 5 \$ par élève au tournant du siècle. En 1906, les commissaires le portent à 6,25 \$ par élève; ils avouent alors que les religieuses reçoivent ce montant et qu'il n'y a " aucun motif pour que l'allocation des institutrices laïques soit basée sur un chiffre moindre ${ }^{15}$. Dans bien des cas, toutefois, ces augmentations ne suffisent pas à résoudre les problèmes financiers qui assaillent les directrices. Pour certaines, une seule solution s'impose : fermer l'école - Joséphine Dorval a dû s'y résoudre en 1898, après que les commissaires eurent refusé de lui venir en aide en lui versant, en sus de son octroi, une somme additionnelle de $100 \${ }^{16}$. 


\begin{tabular}{|c|c|c|c|c|}
\hline \multicolumn{5}{|c|}{$\begin{array}{l}\text { Répartition des dépenses contractées par la Commission scolaire catholique de } \\
\text { Montréal pour le soutien des écoles en } 1880-1881 \text { et en 1906-1907 }\end{array}$} \\
\hline \multirow[b]{2}{*}{ Catégories d'écoles } & \multicolumn{2}{|c|}{$1880-1881$} & \multicolumn{2}{|c|}{$1906-1907$} \\
\hline & Nombre & $\begin{array}{l}\text { Dépenses pour } \\
\text { ces écoles en \$ }\end{array}$ & Nombre & $\begin{array}{l}\text { Dépenses pour } \\
\text { ces écoles en } \$\end{array}$ \\
\hline $\begin{array}{l}\text { Écoles laïques de garçons } \\
\text { " sous contrôle " des commissaires }\end{array}$ & 6 & 40917.68 & 8 & 102635.11 \\
\hline Écoles de frères subventionnées & 2 & 3841.29 & 8 & 44134.91 \\
\hline Écoles de soeurs subventionnées & 7 & 3540.25 & $22^{*}$ & 70171.54 \\
\hline Écoles des " dames et demoiselles" & 10 & 3421.42 & 12 & 17283.10 \\
\hline
\end{tabular}

* Incluant les écoles dites spéciales.

Sources: Rapport financier des commissaires d'écoles catholiques romains de la Cité de Montréal, 1880-1881, cédule $B$ et Rapport financier de la Commission des écoles catholiques de Montréal pour l'année scolaire 1906-1907, cédules $A, B, C, D$.

Fonctionnant avec des moyens précaires, les écoles des " dames et demoiselles » demeurent, au tournant du siècle, les moins coûteuses de toutes celles soutenues par la Commission scolaire (voir tableau 1). Les écoles laïques de garçons continuent d'entraîner les plus fortes dépenses. D'autre part, le réseau d'écoles congréganistes subventionnées s'est développé de façon considérable, phénomène qui reflète l'expansion des communautés religieuses depuis la seconde moitié du XIX $\mathrm{X}^{\mathrm{e}}$ siècle. Les écoles de sœurs se sont multipliées le plus rapidement. Ce sont les plus nombreuses désormais et elles accaparent près du tiers des crédits affectés au soutien des écoles. En revanche, les écoles laïques subventionnées ont connu une croissance très lente. En 1905-1906, elles se situent loin derrière celles tenues par les religieuses, tant sur le plan numérique qu’au chapitre des dépenses.

Or, même si elle ne favorise pas leur expansion, la Commission scolaire catholique soumet les écoles des "dames et demoiselles " à une surveillance de plus en plus étroite. Ainsi elle voit à ce que les institutrices qui y sont engagées soient munies d'un brevet d'enseignement, règlement auquel échappent les frères et les sœurs. Selon un rapport soumis en 1881 par la Commission scolaire au Département de l'instruction publique, 22 des 41 femmes oeuvrant alors dans les écoles laïques subventionnées ne possèdent aucun brevet. Les commissaires citent en particulier l'école de Madame Marchand où, cette dernière exceptée, toutes les enseignantes «n'ont pas de diplôme, les unes parce qu'elles n'ont pas l'âge, les autres par négligence ${ }^{17}$. Dix ans plus tard, 32 des 38 laïques œuvrant dans ces mêmes écoles possèdent un brevet. La plupart, soit $90 \%$, l'ont obtenu devant un des bureaux d'examinateurs de la province. Trois directrices possèdent de leur côté un diplôme d'école normale. Enfin, 67 \% de l'ensemble détiennent un brevet d'école élémentaire. Ce profil des enseignantes laïques se distingue de celui des maîtres employés en 1881 dans les écoles laïques de garçons 
appartenant à la Commission. En effet, 67 des 68 instituteurs qui y travaillent sont munis d'un brevet, la moitié l'ayant obtenu dans une école normale. Une minorité d'enseignants, $19 \%$, possèdent un brevet d'école élémentaire, $53 \%$, un brevet d'école modèle et $29 \%$, un brevet d'école académique ${ }^{18}$.

Le niveau de compétence plus élevé des maîtres laisse croire que ces derniers exigent un salaire plus élevé. Un dénombrement effectué en 1881 par le Bureau des commissaires révèle en effet l'existence d'un écart majeur entre les traitements annuels versés aux hommes et ceux accordés aux femmes. Alors que les " principaux " reçoivent entre $1,100 \$$ et $1,500 \$$, les directrices laïques ne gagnent qu'entre $200 \$$ et 250 \$, à l'exception de Madame Marchand qui obtient 300 \$. La majorité des instituteurs reçoivent entre $400 \$$ et 850 \$. Quant aux institutrices, elles sont payées entre 60 \$ et 200 \$ par année, une seule obtenant ce dernier montant. Les enseignantes reçoivent donc des traitements dérisoires qui ne leur permettent guère de vivre de façon décente. Déjà il apparaît que le sexe plus que la compétence détermine les différences dans les salaires versés aux hommes et aux femmes comme le montrent deux exemples tirés du dénombrement effectué en 1891. Phélonise Thibodeau, qui a obtenu en 1863 un brevet d'école modèle de l'École normale Laval et qui dirige une école depuis plus de quinze ans, gagne 250 \$ par année, alors que le "principal " de l'École Champlain, H.-O. Doré, qui a obtenu en 1866 un brevet d'école modèle devant un bureau d'examinateurs, reçoit un salaire de 1,100 \$. Par ailleurs, la Commission scolaire accorde 120 \$ à Athénaïs Bibaud qui a obtenu en 1882 un brevet d'école académique accordé par le bureau des examinateurs de Montréal, tandis qu'il verse cinq fois plus à J.-R. Bellefeuille, un enseignant à l'École Champlain qui, en 1880, a obtenu devant le même bureau un brevet d'école modèle, donc de valeur moindre ${ }^{19}$. Bref, les femmes employées dans les écoles laïques subventionnées reçoivent systématiquement moins que leurs confrères, même si elles possèdent un diplôme de valeur équivalente ou supérieure et ont accumulé plusieurs années d'expérience.

Cela n'empêche pas la Commission scolaire de procéder, au début du $20^{\text {e }}$ siècle, à une évaluation plus serrée de leur compétence. En 1905, elle avise toutes les directrices qu'elles ne recevront leur octroi qu'à la condition de subir avec les mâ̂tresses qu'elles emploient un examen supplémentaire devant son propre bureau spécial d'examinateurs. Jusqu'à présent, seuls les professeurs enseignant dans les écoles laïques de garçons se sont vus imposer cette épreuve. Les directrices protestent aussitôt et demandent d'être dispensées de l'examen. Les commissaires n'accordent cette faveur qu'à celles n'ayant aucune charge d'enseignement. Les résultats de l'examen témoignent de la réticence des écoles laïques subventionnées à se placer sous la tutelle des autorités scolaires publiques. Alors que 24 enseignantes diplômées réussissent l'examen, 21 d'entre elles, dont 4 directrices, refusent de s'y présenter. Les commissaires menacent alors de priver de leur octroi tous les établissements refusant de remplacer les enseignantes fautives par d'autres ayant subi l'examen requis ${ }^{20}$.

Ce pouvoir de contrôle sur les écoles des « dames et demoiselles ", les commissaires entendent l'exercer à d'autres niveaux. Depuis la fin du $19^{\mathrm{e}}$ siècle, ils soumettent ces dernières à des inspections de plus en plus sévères sur les plans pédagogique et hygiénique. Chargé par les commissaires de les visiter, l'inspecteur des écoles catholiques 
de Montréal, Urgel-Eugène Archambault, les critique sévèrement. Celles qui sollicitent une subvention de la Commission scolaire lui apparaissent pour la plupart défectueuses. C'est le cas de l'école mixte dirigée par Madame A. Richer sur la rue Logan qu'Archambault visite en 1886. Celui-ci dénonce alors le "manque trop prononcé d'autorité de la part de la maîtresse ", le local exigu et l'ameublement "des plus anciens et des plus incommodes ", lequel est non seulement contraire aux lois de l'hygiène, " mais aussi aux règles de la morale et de la discipline ». Quelques années plus tard, Archambault frappe du même verdict l'école de Mary McGee, située sur la rue Prince Arthur : "... il y a des garçons de 13 et 14 ans assis sur les mêmes bancs à côté de jeunes filles à peu près du même âge. Ses maîtresses ne paraissent pas soupçonner qu'il peut y avoir de graves inconvénients à cet état de chose. Sur la remarque que j'en ai faite à la Directrice, il m'a été répondu que c'est la coutume aux États-Unis où elle a enseigné plusieurs années ${ }^{21}$. L'inspecteur juge tout aussi sévèrement les écoles laïques déjà subventionnées par le Bureau des commissaires. Dans un rapport soumis en 1885, Archambault dénonce la pauvreté de leur matériel scolaire et l'insalubrité de leurs salles de classe, où les élèves " n'ont pas le quart de l'espace exigé pour se mouvoir et pour respirer ». L'octroi versé par le Bureau étant insuffisant, les directrices accueillent manifestement un trop grand nombre d'élèves, dans le but d'augmenter les revenus provenant de la rétribution mensuelle ${ }^{22}$. Des plaintes contre les écoles des «dames et demoiselles » émanent aussi d'autres milieux. En 1895, par exemple, plusieurs parents mécontents accusent l'école tenue par Ida Labelle d'enfreindre les lois de l'hygiène en accueillant près de 190 élèves, alors qu'elle ne peut qu'en recevoir 80 environ $^{23}$.

En réponse aux critiques qui lui parviennent, la Commission scolaire effectue, la même année, une inspection globale des écoles laïques subventionnées au point de vue de leur emplacement, de l'hygiène et de l'enseignement, afin de déterminer s'il y a lieu de les supprimer ou non. Elle décide alors de retirer l'octroi à sept d'entre elles, alléguant que « les enfants qui les fréquentent peuvent facilement trouver place dans les écoles avoisinantes ${ }^{24}$. Ce geste radical soulève un tollé de protestations au sein de plusieurs paroisses desservies par les écoles touchées. Des requêtes portant la signature de parents, du curé et de contribuables influents sont adressées aux commissaires et réclament le maintien de la subvention et, donc, des écoles. Devant cette levée de boucliers, la Commission consent à en récupérer quatre. Elle avise toutefois les directrices qu'elles ne pourront plus accueillir dans leurs classes des garçons de plus de huit $a^{25} s^{25}$ Ce règlement, qui découle d'une condamnation de la mixité, avant tout pour des motifs d'ordre moral, ne peut qu'aggraver les difficultés financières de ces femmes qui dépendent largement de la rétribution mensuelle pour rencontrer leurs dépenses.

Cette offensive perpétrée contre les écoles laïques subventionnées préfigure la ligne de conduite que la Commission scolaire catholique va désormais adopter à leur endroit. Elle tient à leur imposer ses propres règlements, afin de les aligner sur les écoles laïques de garçons directement sous sa gouverne. L'inspecteur des écoles catholiques de Montréal encourage ce mouvement, de même que des commissaires réformistes pour la plupart d'allégeance libérale - qui favorisent, à la suite du premier, l'expansion d'un système scolaire public administré par une commission aux pouvoirs accrus. 
D'autre part, subissant l'influence des réformistes, la Commission scolaire manifeste au début du siècle un intérêt nouveau pour l'enseignement féminin qu'elle désire maintenant organiser et prendre en main. En 1908, elle décrète que l'éducation des filles doit être "mise sur le même pied que l'éducation des garçons quant à l'efficacité et quant au coût de l'éducation pour les parents ». Cette préoccupation pour l'instruction des filles se nourrit d'une valeur maîtresse, la famille, pilier de la société, dont l'industrialisation et 1'urbanisation semblent menacer l'harmonie et la stabilité. En effet, les commissaires désirent avant tout préparer les filles à assumer leur vocation " naturelle » de gardiennes du foyer, donc de l'ordre social. Aussi entendent-ils leur donner des cours d'enseignement ménager, grâce auxquels elles deviendront de parfaites maîtresses de maison. Une résolution rendant ce dernier obligatoire est adoptée par la Commission en $1909^{26}$. Enfin, le souci de développer l'enseignement féminin implique, sur le plan matériel, la construction d'écoles de filles. Une séparation complète des sexes, telle qu'elle existe déjà dans les écoles congréganistes subventionnées, sera alors réalisée.

La réunion de tous ces facteurs va entraîner l'élimination graduelle des écoles des "dames et demoiselles » durant la première décennie du $20^{\text {e }}$ siècle. S'appuyant sur des rapports de plus en plus fréquents qui insistent sur leurs multiples défectuosités aux points de vue matériel, disciplinaire et pédagogique, la Commission scolaire procède à de nouvelles fermetures. Pendant ce temps, les premières écoles de filles construites aux frais de la Commission ouvrent leurs portes. Les trois premières ${ }^{27}$ sont confiées aux soeurs de la Congrégation de Notre-Dame. Cependant, les commissaires comptent aussi recevoir les filles dans des écoles dirigées par des laïques. La mort de Malvina Marchand en 1906 va leur en fournir l'occasion. À la suite des démarches effectuées par la nouvelle directrice, Athénaïs Bibaud, ils acceptent de placer son école sous leur autorité immédiate. Ils décident ensuite de construire un nouvel édifice portant, à la demande de Bibaud, le nom d'Académie Marchand. "C'est un progrès que nous signalons avec bonheur ", déclarent les commissaires. "Vienne vite le jour où toutes nos institutrices laïques seront dans des écoles bâties par la Commission ! Ce sera la fin de l'enseignement donné dans des maisons privées transformées en écoles, mais non construites à cette fin $"^{28}$.

L'inauguration de l'Académie Marchand, qui a lieu en 1910, marque effectivement la fin d'une époque. La même année, la Commission scolaire annonce la construction d'une nouvelle école laïque de filles « qui remplacera plusieurs petites écoles privées dans lesquelles enseignent des institutrices laïques ... ${ }^{29}$. L'ouverture de l'École Garneau en 1911 entraîne, de fait, la fermeture de six écoles laïques subventionnées. Les commissaires signalent toutefois que la plupart des institutrices enseignant dans l'une ou l'autre de ces écoles sont devenues titulaires d'une classe. Une ancienne directrice, Maria Manger, est placée à la tête du nouvel établissement. Seulement deux maisons subventionnées survivent au naufrage grâce à l'appui de quelques commissaires ${ }^{30}$.

Le nombre d'écoles de filles appartenant à la Commission se multiplie rapidement au cours des années suivantes. On en compte 11 en 1915. Deux seules ont des laïques à leur tête - l'Académie Marchand et l'École Garneau - toutes les autres étant dirigées 
par des congrégations enseignantes. Également propriétaires de dix-sept écoles de filles subventionnées, les religieuses ont désormais la haute main sur les écoles primaires catholiques de filles de Montréal. Des enseignantes laïques continuent cependant d'assurer la direction d'écoles mixtes. Elles en dirigent cinq en 1915. Deux sont la propriété de la CECM; les trois autres en reçoivent une subvention. Par ailleurs, sur un total de 124 institutrices laïques régulièrement employées dans les écoles relevant de la CECM, 50 enseignent dans des établissements dirigés par des laïques, 13 dans des écoles confiées à un principal laïc, 6 dans des écoles de frères et 55 dans des écoles tenues par des religieuses ${ }^{31}$.

Ainsi, le déclin des écoles "des dames et des demoiselles » et l'intégration croissante des laïques s'accompagnent d'une diminution de leurs sphères d'influence et d'autorité dans le champ de l'enseignement primaire montréalais. De fait, en 1915, les enseignantes laïques n’ont la responsabilité que de 7 des 61 écoles relevant de la Commission et la plupart enseignent dans des maisons dirigées par des hommes et, surtout, par des religieuses.

Certes, leur situation s'est améliorée au point de vue financier. En 1910, les commissaires adoptent une échelle de traitements annuels pour les directrices et les institutrices laïques employées dans les écoles laïques et dans les écoles congréganistes. Un minimum de 700 \$ et un maximum de 1,000 \$ sont prévus pour les premières; un minimum allant de 350 à 500 \&et un maximum allant de 500 à $700 \$$ sont fixés pour les secondes, leur salaire étant établi d'après le niveau du diplôme obtenu. Cependant, les directrices reçoivent considérablement moins que leurs homologues masculins, auxquels on accorde 1,200\$ à 1,800\$. De leur côté, les instituteurs se voient attribuer un minimum de $700 \$$ et un maximum s'échelonnant de 1,200 à 1,500 \$. Les enseignantes reçoivent donc moins que la moitié du salaire versé à leurs confrères $^{32}$. Il s'agit d'une inégalité de traitement importante qui durera encore plusieurs décennies.

\section{Conclusion}

Cette étude de cas révèle qu’à Montréal au XIX siècle, la participation des femmes laïques francophones à l'enseignement se situe dans un cadre d'exercice particulier dont nous avons tenté de retracer l'origine et l'évolution. Ce cadre d'exercice prend forme avec l'arrivée dans la cité de nombreuses immigrantes britanniques qui y implantent le modèle de l'enseignante laïque à la tête d'écoles indépendantes ou semiindépendantes. Les francophones, des femmes bien souvent, nous l'avons constaté, empruntent ce modèle qui va perdurer même après la création et l'expansion de la CECM. Ces femmes sont venues à l'enseignement pour des motifs variés, à commencer par le besoin de gagner leur vie. Dans certains cas, on voit même leurs écoles fonctionner selon le modèle de l'entreprise familiale. Par ailleurs, on dénote chez plusieurs enseignantes la volonté de mettre avantageusement à profit leurs connaissances et d'améliorer leurs compétences. Certaines, telles Mesdames Trudeau et Marchand, feront même carrière dans l'enseignement et se tailleront une solide réputation dans leur milieu. Enfin, ce cadre d'exercice aura aussi permis à des femmes d'assumer 
la direction et la gestion d'écoles qui portent d'ailleurs leur nom. En somme, on peut penser que ces enseignantes ont trouvé dans leur travail une source importante d'accomplissement. D'autre part, des facteurs externes favorisent leur présence dans l'enseignement : l'accroissement démographique de Montréal, la demande accrue d'instruction primaire venant des parents et le refus persistant de la CECM d'investir autant dans l'éducation des filles que dans celle des garçons. Être à la tête d'écoles indépendantes ou semi-indépendantes représente pour les enseignantes un défi de taille : l'instabilité, la précarité, l'insécurité financière et des conditions difficiles de travail caractérisent en effet bien souvent ces écoles.

Le début du $\mathrm{XX}^{\mathrm{e}}$ siècle voit un effacement important du modèle d'enseignement laïque francophone établi presque un siècle plus tôt. Car la CECM soumet les écoles "de dames et demoiselles " à une surveillance de plus en plus étroite, alors même qu'elle manifeste un intérêt nouveau pour l'éducation des filles qu'elle entend, à l'évidence, confier aux communautés religieuses. La grande majorité des enseignantes laïques francophones sont désormais intégrées dans le réseau scolaire public. Cette intégration entraîne certes une amélioration de leurs conditions de travail. Mais elle signifie en même temps pour ces femmes une perte d'autonomie et leur confinement aux rangs inférieurs de la hiérarchie enseignante. Il n'en demeure pas moins que leur contribution à l'enseignement, d'une longévité pour le moins étonnante même dans ce contexte, constitue une dimension incontournable de l'œuvre éducative accomplie auprès de la jeunesse montréalaise.

\section{Notes}

1 "École Marchand ", dans Nos écoles läques, 1846-1946, Album-souvenir (Montréal : 1947), 57.

2 Montréal compte 57715 habitants en 1852; en 1861, elle en compte 90323.

3 Abbé Louis-Adélard Desrosiers, "Chronique de la Commission des écoles catholiques de Montréal ", dans Nos écoles lä̈ques, 9-13.

$4 \quad$ Notice sur les écoles relevant du Bureau des commissaires catholiques romains de la cité de Montréal (Montréal : s. é., 1886), 7.

5 La Notice de 1886 ne rapporte qu'une exception: l'École de Phélonise Thibodeau, située sur la rue Cadieux. Voir Notice sur les écoles, 87.

6 Voir les données publiées dans la Notice sur les écoles, tableau B, non paginé.

7 Marta Danylewycz, "Sexes et classes sociales ", dans Nadia Fahmy-Eid et Micheline Dumont, Maîtresses de maison, maîtresses d'école. Femmes, famille et éducation dans l'histoire du Québec (Montréal : Les Éditions du Boréal Express, 1983), 112-113.

8 "À la mémoire de Madame Marchand ", Revue canadienne, 52 (février 1907), 200.

9 Archives de la Commission des écoles catholiques de Montréal (dorénavant ACECM), Rapport du mois de janvier des commissaires d'écoles de la municipalité de Montréal, 18901891, dossier Fonds de pension.

10 Urgel-Eugène Archambault à Mademoiselle Élisa DesRivières, 14 juillet 1890, ACECM, Correspondance générale de Urgel-Eugène Archambault, Registre B.

11 Notice sur les écoles relevant du Bureau des commissaires catholiques romains de la cité $\mathrm{de}$ Montréal (Montréal : s. é., 1893), 22, 24-25.

12 C'est-à-dire appartenant à la Commission scolaire catholique. 
13 ACECM. Rapport financier des commissaires d'écoles catholiques romains de la cité de Montréal, 1880-1881, cédule B.

14 Notice sur les écoles, 1893, 24-25.

15 ACECM, Registre des délibérations de la Commission des écoles catholiques de Montréal, vol. 6 (25 septembre 1906).

16 ACECM, Registre des délibérations, vol. 4 (11 janvier, 8 février et 8 mars 1898).

17 Municipalité scolaire de la cité de Montréal, Rapport semestriel supplémentaire pour 1881 (30 juin 1881), ACECM, dossier Fonds de pension.

18 Rapport du mois de janvier des commissaires d'écoles de la municipalité de Montréal, 18901891, ACECM, dossier Fonds de pension.

19 Ibid.

20 ACECM, Registre des délibérations, vol. 6 (14 et 28 février 1905, 27 juin 1905).

21 U.-E. Archambault aux commissaires d'écoles catholiques romains, 10 novembre 1886, ACECM, Correspondance générale, Registres A et B, 2 octobre 1891.

22 Id. à id; 21 janvier 1885, ACECM, dossier Écoles subventionnées, 1874-1970.

23 ACECM, Registre des délibérations, vol. 4 (14 janvier 1896).

24 ACECM, Registre des délibérations, vol. 4 (10 décembre 1895, 14 et 28 janvier 1896).

25 Ibid., vol. 4 (10 décembre 1895, 14 et 28 janvier 1896.

26 Ibid., vol. 8 (27 octobre 1908, 8 juin 1909).

27 Il s'agit des écoles Saint-Eusèbe et Sainte-Hélène et de l'Académie Jeanne Leber.

28 ACECM, Rapport financier de la Commission des écoles catholiques de Montréal, 1908-1909, 5.

29 ACECM, Rapport financier de la Commission des écoles catholiques de Montréal, 1910-1911, 4-5.

30 Ibid., 1910-1911, 4-5. Les deux écoles qui continuent de recevoir un octroi sont l'école de Madame MacKay-Wolff et l'école de Mademoiselle Elodie Viger.

31 Données tirées de la Notice sur les écoles administrées par la Commission des écoles catholiques de Montréal (Montréal : s. é, 1915), 27-29, 86-89.

32 Voir ibid., 435-446.

\section{ERRATA}

Dans le numéro d'automne 2019 (vol 31, no 2), des erreurs relatives aux notes de bas de pages se sont glissées. Veuillez donc noter les modifications suivantes. Ces erreurs ont été corrigées dans la version disponible en ligne de la Revue d'histoire de l'éducation.

- À la page 85 , à la fin de la citation de la ligne 6 du paragraphe débutant à la mi-page, on doit trouver la note suivante : ACECM, Registre des délibérations, vol. 4 (10 décembre 1895,14 et 28 janvier 1896).

- À la page 86, à la fin de la citation de la 4e ligne, 3e paragraphe, on doit trouver la note suivante : ACECM, Rapport financier de la Commission des écoles catholiques de Montréal, 1910-1911, 4-5.

Merci de votre compréhension. 